PAPER

\title{
Long term visual and neurological prognosis in patients with treated and untreated cavernous sinus aneurysms
}

\author{
N Goldenberg-Cohen, C Curry, N R Miller, R J Tamargo, K P J Murphy
}

J Neurol Neurosurg Psychiatry 2004;75:863-867. doi: 10.1136/jnnp.2003.020917

See end of article for
authors' affiliations
$\ldots \ldots \ldots \ldots \ldots \ldots \ldots \ldots . \ldots . \ldots$.
Correspondence to:
Dr N R Miller, Wilmer
Ophthalmological Institute,
Johns Hopkins Hospital,
600 N Wolfe Street,
Maumenee B-109,
Baltimore, MD 21287
9204, USA; nrmiller@
ihmi.edu
Received 8 July 2003
In revised form
28 October 2003
Accepted
7 November 2003

See end of article for authors' affiliations

Correspondence to: Ophthalmolón Johns Hopkins Hospital, Maumen B-109,

Baltimore, MD 21287

9204, USA; nrmiller@

\begin{abstract}
Objective: To determine the long term visual and neurological outcome of patients diagnosed with cavernous sinus aneurysms (CCAs).

Methods: Prospective follow up for at least five years or until death of 31 retrospectively recruited patients (27 women, 4 men) with treated and untreated CCAs.

Results: There were 40 aneurysms in all. Mean age at diagnosis was 60.4 years (range 25 to 86 ; median 64). The most common symptoms were diplopia (61\%), headache (53\%), and facial or orbital pain (32\%). Fiffeen patients $(48 \%)$ were diagnosed after they developed cranial nerve pareses, four $(13 \%)$ after they developed carotid-cavernous sinus fistulas (CCFs), and $12(39 \%)$ by neuroimaging studies done for unrelated symptoms. Twenty one patients (68\%) had treatment to exclude the aneurysm from circulation, 10 shortly after diagnosis and 11 after worsening symptoms. Immediate complications of treatment occurred in six patients and included neurological impairment, acute ophthalmoparesis, and visual loss. Ten patients (32\%) were observed without intervention. Over a mean (SD) follow up period of 11.8 (7.7) years, eight had improvement in symptoms, five remained stable, and eight deteriorated. Among the 10 patients followed without intervention, none improved spontaneously, three remained stable, and seven worsened.

Conclusions: Most treated patients in this series improved or remained stable after treatment, but none improved without treatment. The long term prognosis for treated cases is relatively good, with most complications occurring immediately after the procedure. Endovascular surgery has decreased the morbidity and mortality of treatment so should be considered for any patient with a CCA.
\end{abstract}

A neurysms arising from the cavernous portion of the internal carotid artery (ICA) may produce a variety of neurological deficits, primarily those related to vision, including diplopia from single or multiple oculomotor nerve pareses, decreased visual acuity from compressive or ischaemic optic neuropathy, corneal and facial anaesthesia or hypaesthesia from involvement of the trigeminal nerve, and facial pain. ${ }^{12}$ Like other intracranial aneurysms, these aneurysms can rupture, but this is a rare event and when it occurs it usually does not produce a subarachnoid or intracerebral haemorrhage because of the cavernous location of the aneurysm. ${ }^{3}$ Instead, rupture of a cavernous carotid aneurysm (CCA) usually causes a carotid-cavernous sinus fistula or, rarely, epistaxis. ${ }^{3}$

Although most intracranial (intradural) aneurysms can be treated surgically or with endovascular techniques that isolate them from the parent vessel without occluding that vessel, CCAs usually cannot be treated in these ways. ${ }^{4}$ In general, the treatment of CCAs requires occlusion of the ipsilateral ICA with its attendant risks of stroke, blindness, or both. ${ }^{56}$ Endovascular occlusion of the ipsilateral ICA is said to have lower risks of subsequent aneurysm rupture and cerebral ischaemic complications than carotid ligation, ${ }^{6-8}$ but this issue remains controversial. Thus, although some investigators advocate treatment of both symptomatic and asymptomatic CCAs, ${ }^{49}$ others recommend no treatment. ${ }^{3}{ }^{10}$ The reason for this controversy relates to a lack of information on the long term natural history of these aneurysms, as well as on the long term results of treatment. ${ }^{8}$ Our aim in this study was to determine the long term $(\geqslant 5$ years) visual and neurological status of patients with both treated and untreated CCAs.

\section{METHODS}

After approval from the institutional review board of the Johns Hopkins Hospital, we searched the files of the Neuroophthalmology Unit of the Wilmer Eye Institute and the aneurysm database of the Division of Neuroradiology of the Johns Hopkins Hospital for all patients diagnosed with CCAs between 1967 and 1997. Forty five patients were identified and their case records reviewed. Patients then were contacted and asked to participate in the study. If they agreed to do so, they were asked to complete a questionnaire either by phone or by mail, to update their current state of health, and to provide the names of their physicians, including any neurologists, ophthalmologists, or optometrists who had recently examined them. All patients who agreed to provide this information also signed a request for patient records which was sent to all their physicians. Patient information was updated by re-examination, contact with primary care physicians and other specialists, and contact with family relatives in case of death.

The information obtained in the above manner was reviewed and the following data extracted: age at diagnosis, at time of treatment (if any) and at present; sex; race; past and current medical status; location, size, and shape of the CCA; presence or absence of other aneurysms; previous and current neurological and visual symptoms and signs; treatment; and post-treatment complications.

For purposes of analysis, patients were considered to have experienced improvement if a recent examination showed resolution of one or more oculomotor nerve pareses,

Abbreviations: CCA, cavernous sinus aneurysm; CCF, carotidcavernous sinus fistula; ICA, internal carotid artery 
improvement in ocular alignment by at least 5 prism dioptres, resolution of a trigeminal sensory neuropathy, or if, by history, the patient had resolution or reduction of facial pain or headache or reduction in diplopia. Patients were considered to have worsened if a recent examination revealed development of one or more new oculomotor nerve pareses, worsening of ocular alignment by at least 5 prism dioptres, a trigeminal neuropathy ipsilateral to the aneurysm, or if, by history, the patient had developed facial pain, headache, or new onset of diplopia.

\section{RESULTS}

\section{Patient characteristics}

Of the 45 patients identified as being eligible for the study, we were able to contact 32 or their families $(71 \%)$; of those contacted, 29 patients or their families (91\%) agreed to participate. The remaining 13 patients could not be located, but two had a documented follow up longer than five years. Thus, follow up until death or of at least five years was possible for 31 patients (table 1). Nineteen of these 31 patients $(61 \%)$ were still alive, while 12 had died. None of the deaths was related to the CCA or its treatment, although one patient had died from rupture of a separate intracranial aneurysm. Three patients had died before five years of follow up. Among the remaining 28 patients, the follow up period ranged from five to 35 years, with a mean (SD) follow up of 12.8 (7.4) years, and a median of 10.7 years.

\section{Baseline data}

The baseline characteristics of the 31 patients in this study are shown in table 1 . There was a majority of middle aged white women. The only consistent underlying systemic disorder was hypertension. Twenty two of the 31 patients (71\%) had a unilateral CCA, while nine had bilateral CCAs, making a total of 40 CCAs (table 2). Seventeen of the 22 unilateral CCAs $(77 \%)$ were on the right side. Seven CCAs were considered giant $(>2.5 \mathrm{~cm})$, and four were defined as large $(1.0-2.5 \mathrm{~cm})$. Thirteen patients $(42 \%)$ had seven intracranial aneurysms at locations other than in the cavernous sinus, including the vertebrobasilar arterial region (1), middle cerebral artery (2), anterior communicating artery (1), posterior communicating artery (1), basilar artery (1), and posterior inferior cerebellar artery (1). In addition, three patients had aneurysms of the abdominal aorta.

Nineteen of the 31 patients $(61 \%)$ were symptomatic from their CCAs. The most frequent symptom, present in all 19 of these patients, was double vision (table 1). Seven patients complained of horizontal diplopia, whereas two had vertical diplopia and one had oblique diplopia. The nature of the diplopia in the remaining nine patients was not specified. In

Table 2 Aneurysm characterisation

\begin{tabular}{llll}
\hline Size & $\begin{array}{l}\text { Total } \\
(\mathbf{n}=\mathbf{3 1})\end{array}$ & $\begin{array}{l}\text { Observation } \\
(\mathbf{n}=10)\end{array}$ & Surgery $(\mathbf{n}=\mathbf{2 1})$ \\
\hline Giant $(>2.5 \mathrm{~cm})$ & 7 & 1 & 6 \\
Large $(1-2.5 \mathrm{~cm})$ & 4 & 1 & 3 \\
Laterality & & \\
$\quad \begin{array}{l}\text { Unilateral } \\
\text { Right side } \\
\text { Left side }\end{array}$ & 22 & 5 & 17 \\
Bilateral & 5 & 4 & 13 \\
\hline & 9 & 5 & 4 \\
& & &
\end{tabular}

Table 1 Demographic and clinical characteristics

\begin{tabular}{|c|c|c|c|}
\hline & \multicolumn{3}{|c|}{ Number of patients } \\
\hline & Total $(n=31)$ & Observation $(n=10)$ & Surgery $(n=21)$ \\
\hline Male & $4(13 \%)$ & $2(50 \%)^{*}$ & $2(50 \%)^{*}$ \\
\hline Female & $27(87 \%)$ & $8(30 \%)$ & $19(70 \%)$ \\
\hline White & $29(94 \%)$ & $9(31 \%)$ & $20(69 \%)$ \\
\hline African-American & $2(6 \%)$ & $1(50 \%)$ & $1(50 \%)$ \\
\hline Hypertension & $14(45 \%)$ & $5(36 \%)$ & $9(64 \%)$ \\
\hline Age at diagnosis (years) (mean (SD)) & $60.4(12.2)$ & $60.8(10)$ & $60.2(13.3)$ \\
\hline Age at diagnosis, median (range) & $64(25$ to 86$)$ & $62(40$ to 78$)$ & $65(25$ to 86$)$ \\
\hline \multicolumn{4}{|c|}{ Time to diagnosis (symptomatic patients, $n=19$ ) } \\
\hline Immediate & \multicolumn{3}{|l|}{$\begin{array}{l}11 \\
8\end{array}$} \\
\hline Delayed (<1 year to 14 years) & \multicolumn{3}{|l|}{8} \\
\hline Symptoms & $19(61 \%)$ & $5(26 \%)$ & $14(74 \%)$ \\
\hline Diplopia & $19(100 \%)$ & $5(26 \%)$ & $14(74 \%)$ \\
\hline Retrobulbar pain (ipsilateral) & $7(37 \%)$ & $1(14 \%)$ & $6(86 \%)$ \\
\hline Facial pain (ipsilateral) & $3(16 \%)$ & 0 & $3(100 \%)$ \\
\hline Headache (non-specific) & $10(53 \%)$ & $1(10 \%)$ & $9(90 \%)$ \\
\hline Photophobia & $7(37 \%)$ & 0 & $7(100 \%)$ \\
\hline Asymptomatic & $12(39 \%)$ & $5(42 \%)$ & $7(58 \%)$ \\
\hline Aneurysms at other locations $(n=13)$ & $7(58 \%)$ & $2(29 \%)$ & $5(71 \%)$ \\
\hline Unrelated blurred vision & $2(17 \%)$ & 0 & $2(100 \%)$ \\
\hline \multicolumn{4}{|l|}{ Signs } \\
\hline Ophthalmoparesis & $23(74 \%)$ & $5(22 \%)$ & $18(78 \%)$ \\
\hline Isolated OMNP† & $15(65 \%)$ & $3(20 \%)$ & $12(80 \%)$ \\
\hline III & 6 & 2 & 4 \\
\hline IV & 1 & 0 & 1 \\
\hline $\mathrm{VI}$ & 8 & 1 & 6 \\
\hline Multiple OMNPs & $8(35 \%)$ & $2(25 \%)$ & $6(75 \%)$ \\
\hline Trigeminal sensory neuropathy & $7(30 \%)$ & $1(14 \%)$ & $6(86 \%)$ \\
\hline
\end{tabular}


addition, seven patients had ipsilateral retrobulbar pain, and three had ipsilateral facial pain.

The most common clinical sign at presentation, found in 23 patients $(74 \%)$, was ophthalmoparesis (table 1 ). In 15 patients, this was caused by an isolated abducens (8), oculomotor (6), or trochlear (1) nerve paresis, while in eight patients, it was caused by multiple oculomotor nerve pareses. Two of the 15 patients with an isolated oculomotor nerve paresis and five of the eight patients with multiple pareses also had evidence of an ipsilateral trigeminal sensory neuropathy. No patient had an isolated trigeminal sensory neuropathy, and none had evidence of an optic neuropathy. Four patients with giant or large CCAs had mild ipsilateral proptosis.

The diagnosis of CCAs in the 19 symptomatic patients was made immediately after onset of symptoms in 11 patients $(58 \%)$. In the remaining eight patients, the progression of single or multiple oculomotor nerve pareses led to the eventual diagnosis of the CCA, although in two of these patients, neuroimaging studies had been done shortly after onset of symptoms but had failed to identify the aneurysm. Among the 12 patients whose aneurysms were asymptomatic at the time of diagnosis, four were diagnosed during an assessment for a symptomatic unruptured aneurysm in another location, one after rupture of an aneurysm in another location, one after an unrelated stroke, two because of unrelated decreased vision, and the remaining four during assessment for other symptoms, such as headache, that were thought to be unrelated to the aneurysm.

\section{Treatment}

Twenty one of the 31 patients $(68 \%)$ underwent treatment of their CCAs (table 3 ). Ten of these were treated shortly after diagnosis, while 11 were treated because of worsening of symptoms and signs (10) or because of lack of improvement and new onset of severe headache (1). For the latter group, the time from diagnosis to treatment ranged from less than one year to 14 years.

Among the 21 patients who were treated, 16 (76\%) underwent endovascular occlusion of the ipsilateral ICA. In 15 of these patients, a detachable balloon was used; the remaining patient had detachable platinum coils. Of the six patients treated surgically, two had occlusion of the ipsilateral ICA in the neck, two had surgical trapping of the aneurysm, and one had clipping of the aneurysm within the cavernous sinus. Single photon emission computed tomographic (SPECT) scanning combined with cerebral angiography was done in most of the patients who underwent treatment of their aneurysms, and one patient had an extracranial-intracranial bypass procedure before occlusion of the ipsilateral ICA. Ten patients with CCAs were not treated during the follow up period. Five of these had asymptomatic aneurysms, while five had diplopia with or without ipsilateral facial pain.

\section{Follow up}

Among the 21 patients who were treated, six (29\%) had postoperative complications. Three patients who underwent endovascular treatment experienced neurological deficits. In two, the deficits were transient. The third patient developed a permanent contralateral hemiparesis. Two patients who underwent surgical trapping of their CCA experienced an acute ipsilateral ophthalmoparesis, and one patient who underwent surgical occlusion of the ICA in the neck developed ipsilateral blindness. During a mean (SD) follow up period of 11.8 (7.7) years, median 10.2 years, eight treated patients experienced improvement in signs, symptoms or both, five remained stable, and eight worsened. Two patients subsequently developed a CCA on the opposite side to the treated aneurysm, seven and 19 years after surgical occlusion of the ICA in the neck. Another patient with bilateral CCAs who underwent treatment of one of the aneurysms by endovascular coiling developed a contralateral carotidcavernous sinus fistula four months after treatment. We were unable to identify any factors that were common to patients who experienced postoperative complications and those who had no complications following treatment.

Among the 10 patients who were observed without intervention, four died of unrelated causes shortly after diagnosis. There was no evidence that they had experienced any change in their symptoms or signs. Of the six remaining patients, none experienced improvement in symptoms or signs during the follow up period (two remained stable and four worsened). Significant enlargement of a CCA was reported in one patient. No partial or complete spontaneous closure of the aneurysm was identified.

\section{DISCUSSION}

We identified 31 patients with 40 CCAs, all of whom had follow up of at least five years or until death. Using the PubMed database, we were able to find only a few previous large series of patients with CCAs, but these focused on the natural course of the disease ${ }^{3}$ or did not include long term follow up data after treatment. ${ }^{4}{ }^{11}$ Indeed, a meta-analysis of existing studies done by van der Schaaf et al ${ }^{8}$ could not determine a consistent long term outcome for patients with these lesions. This is not surprising because many patients are lost to follow up after diagnosis in one institution and treatment at another. ${ }^{12}$ All of the patients in our series were treated at our institution and many continue to be followed here as well.

In this study, $87 \%$ of the patients were women, with a mean age at diagnosis of 60.4 years. These data are consistent with those of other studies, showing that CCAs mainly affect older women. ${ }^{10}{ }^{12}{ }^{13}$ Similarly, hypertension is a known risk factor for the development of intracranial aneurysms, and 14 of the 24 patients $(58 \%)$ for whom a full medical history was available had hypertension at the time of the diagnosis of their aneurysm.

The presence of bilateral CCAs in nine of our patients is consistent with the findings of others that bilateral CCAs are not uncommon. ${ }^{14}{ }^{15}$ In addition, several patients had aneurysms in other intracranial and extracranial locations, suggesting an acquired degenerative process secondary to hypertension, as nine of the 13 patients $(69 \%)$ had hypertension or an underlying genetic susceptibility to structural weakness of the vascular wall. The latter seems less likely because we identified only one patient with a family history of intracranial aneurysm, and in that case it was the patient's first cousin who was affected. Finally, none of the patients in this study had documented systemic disorders, such as Ehlers-Danlos syndrome type IV, which are believed to be associated with an increased risk of intracranial aneurysm.

CCAs rarely rupture. Instead, most produce symptoms from a mass effect within the cavernous sinus. It is thus not surprising that the most common symptom in our series, as well as in the series of other investigators, was double vision, ${ }^{11} 12$ with the most common presenting sign being ophthalmoparesis. It is interesting that despite the close association of the oculomotor nerves and the first and second divisions of the trigeminal nerve within the cavernous sinus, only $26 \%$ of the patients in our series had multiple cranial neuropathies involving the trigeminal nerve at the time of diagnosis.

When CCAs rupture, they generally produce a carotidcavernous sinus fistula, the treatment of which is relatively straightforward, with little or no mortality and little 


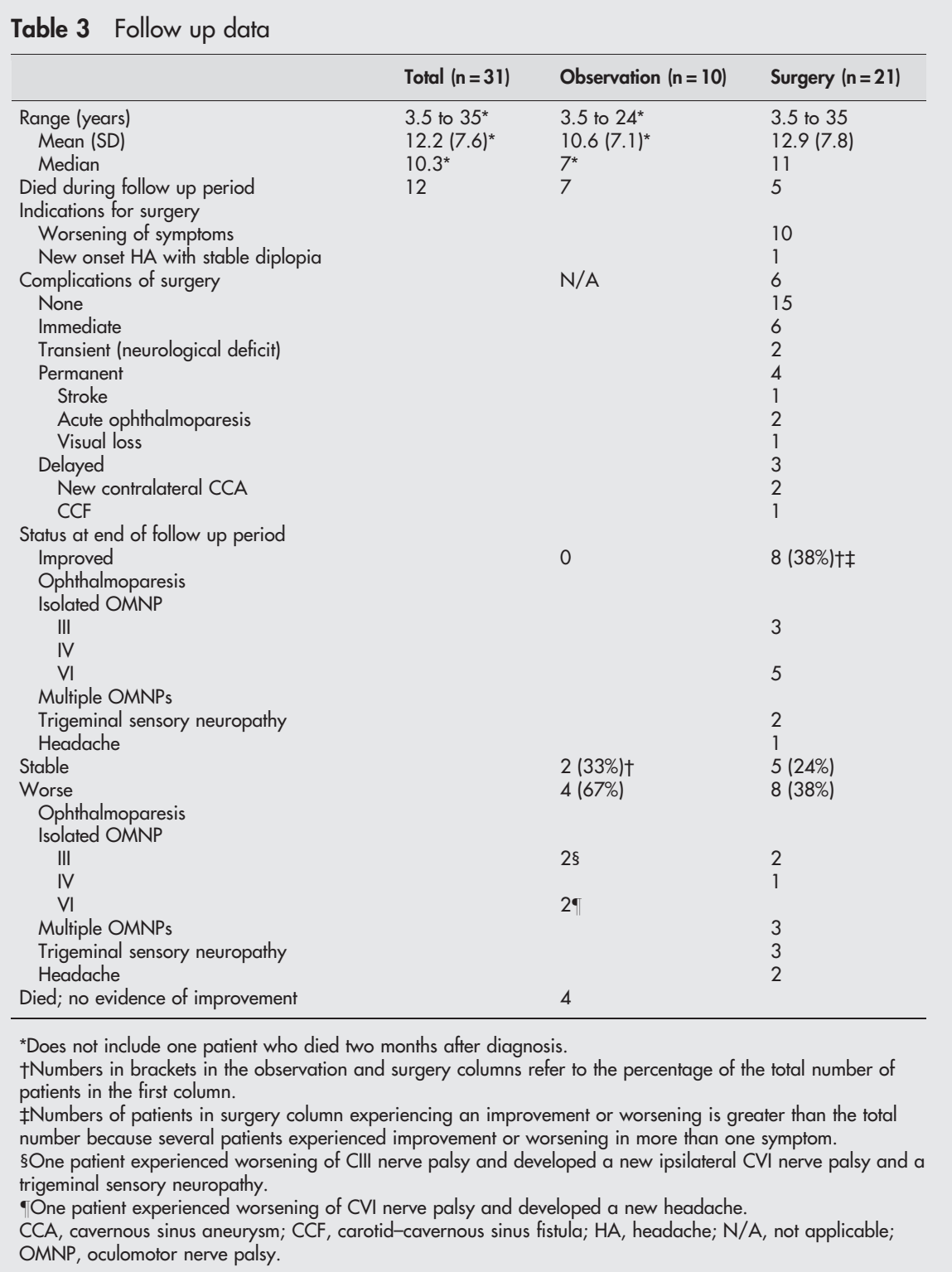

morbidity using current endovascular techniques. ${ }^{49}$ Thus the treatment of unruptured CCAs must balance the natural history of these aneurysms against the potential complications of treatment. Because the natural history of CCAs has been said to be fairly benign, ${ }^{310}$ whereas treatment generally requires occlusion of the parent ICA, with its potential complications, many clinicians have recommended that these lesions should not be treated. ${ }^{3}{ }^{10}$ However, the mortality and morbidity of treatment have decreased significantly in recent years, partly as the result of improved pretreatment screening with techniques such as temporary balloon occlusion combined with SPECT scanning and cerebral angiography, and partly because of improved equipment and techniques for endovascular treatment. ${ }^{4917}$ Most of the patients in our series underwent endovascular treatment of their aneurysms and it thus is not surprising that there was no mortality and little permanent morbidity in the treated patients. Indeed, only one patient who underwent endovascular treatment of a CCA had a permanent neurological deficit. Although some investigators have recommended against occlusion of the ICA to treat a CCA as there is the possibility of developing aneurysms on the opposite side because of increased blood flow, this phenomenon occurred in only two of the 21 treated patients in our series during the follow up period, a finding consistent with that of Bavinzski et al. ${ }^{9}$

We are aware that this study has several inherent weaknesses. In the first place, we were unable to use a validated questionnaire to determine the status of the patients, nor were all patients examined in a standardised fashion. Indeed, in many cases, we had to rely on information supplied by other physicians. In addition, the decision as to whether or not to treat the CCA was individualised for each patient, based on various different criteria. Finally, the number of patients who were not treated and who were still alive at the time of follow up was small. Nevertheless, the fact remains that the majority of symptoms and signs of treated patients improved or remained stable following treatment, whereas the majority of patients who were not treated remained stable or worsened over the follow up period. Improvement of symptoms and signs after treatment has also been reported in previous studies. ${ }^{818} 19$ Thus, when combining the lack of mortality and low permanent morbidity of treatment with the potential for improvement or stabilisation of signs and symptoms, it seems to us that treatment should be considered in every patient with a symptomatic CCA, and 
may even be appropriate for selected patients with asymptomatic CCAs, assuming optimum resources for endovascular therapy.

\section{ACKNOWLEDGEMENTS}

This study received support from the Isabel and Zanvyl Krieger Fund (Baltimore, Maryland).

\section{Authors' affiliations}

N Goldenberg-Cohen, N R Miller, Wilmer Ophthalmological Institute, Johns Hopkins University School of Medicine, Baltimore, Maryland, USA C Curry, Johns Hopkins University School of Medicine

R J Tamargo, Department of Neurosurgery, Johns Hopkins University School of Medicine

K P J Murphy, Department of Radiology, Johns Hopkins University School of Medicine

Competing interests: none declared

Dr Cohen is now associated with the Schneider Children's Medical Centre of Israel, Petah-Tiqva, Israel

\section{REFERENCES}

1 Newman SA. Aneurysms. In: Miller NR, Newman NJ, eds. Walsh \& Hoyt's clinical neuro-ophthalmology. Baltimore: Williams and Wilkins, 1997:2975-3261.

2 Miller NR. Carotid-cavernous sinus fistulas. In: Miller NR, Newman NJ, eds. Welsh \& Hoyt's clinical neuro-ophthalmology. Baltimore: Williams and Wilkins, 1997:3263-322.

3 Kupersmith MJ, Hurst R, Berenstein A, et al. The benign course of cavernous carotid artery aneurysms. J Neurosurg 1992;77:690-3.

4 Higashida RT, Halbach W, Dowd C, et al. Endovascular detachable balloon embolization therapy of cavernous carotid artery aneurysms: results in 87 cases. J Neurosurg 1990;72:857-63.
5 German WJ, Black SP. Cervical ligation for internal carotid aneurysms. An extended follow-up. J Neurosurg 1965;23:572-7.

6 Goddard AJ, Annesley-Williams D, Gholkar A. Endovascular management of unruptured intracranial aneurysms: does outcome justify treatment? J Neurol Neurosurg Psychiatry 2002;72:485-90.

7 Derdeyn CP, Cross DT, Moran CJ, et al. Postprocedure ischemic events after treatment of intracranial aneurysms with Guglielmi detachable coils. J Neurosurg 2002;96:837-43.

8 van der Schaaf IC, Brilstra EH, Buskens E, et al. Endovascular treatment of aneurysms in the cavernous sinus: a systematic review on balloon occlusion of the parent vessel and embolization with coils. Stroke 2002;33:313-18.

9 Bavinzski G, Killer M, Ferraz-Leite H, et al. Endovascular therapy of idiopathic cavernous aneurysms over 11 years. Am J Neuroradiol 1998;19:559-65.

10 Linskey ME, Sekhar LN, Hirsch WL, et al. Aneurysms of the intracavernous carotid artery: natural history and indications for treatment. Neurosurgery 1990;26:933-7, discussion 937-8

11 Kupersmith MJ, Berenstein A, Choi IS, et al. Percutaneous transvascular treatment of giant carotid aneurysms: neuro-ophthalmologic findings. Neurology 1984:34:328-35.

12 Hahn CD, Nicolle DA, Lownie SP, et al. Giant cavernous carotid aneurysms: clinical presentation in fifty-seven cases. J Neuroophthalmol 2000;20:253-8.

13 Trobe JD, Glaser JS, Post JD. Meningiomas and aneurysms of the cavernous sinus. Neuro-ophthalmologic features. Arch Ophthalmol 1978:96:457-67.

14 Linskey ME, Sekhar LN, Hirsch W, et al. Aneurysms of the intracavernous carotid artery: clinical presentation, radiographic features, and pathogenesis. Neurosurgery 1990;26:71-9.

15 Yamada K, Nakahara T, Kishida K, et al. Multiple "mirror" aneurysms involving intracavernous carotid arteries and vertebral arteries: case report. Surg Neurol 2000;54:361-5.

16 Linskey ME, Sekhar LN. Which cavernous sinus aneurysms should be treated? J Neurosurg 1993;78:1008-9.

17 Larson JJ, Tew JM, Tomsick TA et al. Treatment of aneurysms of the internal carotid artery by intravascular balloon occlusion: long-term follow-up of 58 patients. Neurosurgery 1995;36:26-30, discussion 30 .

18 Niiro M, Shimozuru T, Nakamura K, et al. Long-term follow-up study of patients with cavernous sinus aneurysm treated by proximal occlusion. Neurol Med Chir (Tokyo) 2000:40:88-96, discussion 96-7.

19 Kaminogo M, Kitagawa N, Takahata H, et al. Strategy for the treatment of inaccessible unruptured giant and large aneurysms of the internal carotid artery. Neurol Res 2001;23:388-96. 